\title{
Hybrid energy-efficient APTEEN protocol based on ant colony algorithm in wireless sensor network
}

\author{
Jinyu Ma ${ }^{1,2}$, Shubin Wang ${ }^{1 *} \mathbb{D}$, Chen Meng ${ }^{1}$, Yanhong $\mathrm{Ge}^{1}$ and Jingtao Du ${ }^{1}$
}

\begin{abstract}
Due to the limited energy of the sensor nodes, the unreasonable clustering routing algorithm will cause node premature death and low utilization of energy efficiency in wireless sensor network (WSN). In Adaptive Thresholdsensitive Energy Efficient Network (APTEEN), the assignments of the cluster head (CH) are much heavier than other nodes. The $\mathrm{CH}$ unbalanced energy dissipation between nodes that make them die prematurely. Ant colony algorithm can avoid this problem, so this paper presents a double cluster heads Adaptive Threshold-sensitive Energy Efficient Network based on ant colony (ADCAPTEEN). ADCAPTEEN optimizes the cluster head election method compared with APTEEN. It suggests that one master cluster head (MCH) and one vice cluster head $(\mathrm{VCH})$ will be selected in each cluster. The double cluster heads (DCH) can co-work on data collection, fusion, transition, etc. To make routes more stable and energy efficient, this paper proposes a Multiple Adaptive Threshold-sensitive Energy Efficient Network based on Ant-colony (AMAPTEEN). It is the optimization of ADCAPTEEN. And CH selects intermediate node (IM_node) multiple times with ant colony algorithm per round in each cluster, and this way forms multiple route transmission data. Simulation in OPNET proves that compared with APTEEN, ADCAPTEEN reduces energy dissipation, improves node survival rate, and extends network life cycle. AMAPTEEN delays the time of node death, balances energy consumption, and extends network lifetime further operating in the same settings compared with ADCAPTEEN. The proposed two algorithms have good scalability, and they are suitable for large-scale network.
\end{abstract}

Keywords: Wireless sensor network (WSN), APTEEN, Ant colony algorithm, Multiple routes

\section{Introduction}

Wireless sensor networks (WSN) consisting of a large number of sensor nodes are deployed randomly in monitor areas. The applications of the WSN have grown rapidly over the recent years. The performance and the quality of WSN are closely related to the type of routing protocol. Clustering routing protocol commonly used as clustering protocol can effectively facilitates saving energy [1]. Basing on the mode of functioning, WSNs can be classified into proactive, reactive, and hybrid networks [2]. Under proactive network application mode, sensor nodes continuously monitor the surrounding environment and send periodically the monitored data to the user at a constant cycle rate. The reactive network, however, only transmits data when the observed

\footnotetext{
* Correspondence: wangshubin@imu.edu.cn

${ }^{1}$ College of Electronic Information Engineering, Inner Mongolia University,

Hohhot, China

Full list of author information is available at the end of the article
}

variable changes, and the change reaches the conditions that need to be sent. The Low-Energy Adaptive Clustering Hierarchy (LEACH) [3] protocol, one of the early cluster algorithms, belongs to proactive network, but is not suitable for reactive network. The energy-efficient LEACH (EE-LEACH) protocol for data gathering is introduced [4]. It reduces endto-end delay and energy consumption. But nodes which are selected to forward the data to sink node only consider the nodes' residual energy. The improved LEACH selects energy-efficient $\mathrm{CH}$ which considers the energy and distance [5]. The sum of alive nodes is quite improved. The novel energy-efficient clustering mechanism, based on artificial bee colony algorithm, is presented to prolong the network lifetime [6]. Threshold-sensitive Energy Efficient Network (TEEN) belongs to the reactive mode [7]. The proposed Adaptive Threshold-sensitive Energy Efficient Network (APTEEN) [8] is classified as a hybrid network and preserves the advantages of both proactive network and 
reactive network. APTEEN protocol can collect data in a circular way and respond to emergencies. Unfortunately, APTEEN exposes its defects-direct communication between $\mathrm{CH}$ and sink node, which results in high energy consumption and low efficiency. A proposed Hierarchal Multipath Adaptive Threshold-sensitive Energy Efficient Network Protocol (HMAPTEEN) [9] uses selected nodes in each level for routing to the next level. It also allows multiple routes for the information to reach destinations. It improves the routing reliability. But HMAPTEEN selects nodes in each level for routing to the next level causes the energy waste. The proposed Distance Adaptive Threshold Sensitive Energy Efficient Sensor Network (DAPTEEN) [10] enhances the network survivability by removing data redundancy, but only considers the distance between nodes within a cluster regardless of node residual energy.

The APTEEN is a single-cluster head-based protocol with nodes of the same initial energy. The cluster head $(\mathrm{CH})$ needs to perform extra computations which bring about imbalanced node energy consumption and premature death of nodes. Yet, the algorithm based on double cluster heads $(\mathrm{DCH})$, commonly used for LEACH, can eliminate the defects. The proposed double heads static cluster (DHSC) algorithm [11] considers the area density, but does not consider the node residual energy; whereas, a double cluster heads topology control algorithm based on the energy threshold which cater to heterogeneous wireless sensor network (DCCCA) [12] considers those factors but too complicated.

Studies have shown that the design of clustering routing protocols in the WSN introduces DCH or multipath mechanism can reduce the energy consumption of traditional $\mathrm{CH}$ and it can improve network performance. APTEEN is a traditional hybrid clustering routing protocol. In literature [9], it can be seen that HMAPTEEN does not further reduce energy consumption compared to the single-path protocol. Thus, this article makes comparison of APTEEN protocol with double cluster heads Adaptive Thresholdsensitive Energy Efficient Network based on ant colony (ADCAPTEEN) protocol, and ADCAPTEEN protocol with Multiple Adaptive Threshold-sensitive Energy Efficient Network based on ant colony (AMAPTEEN) protocol. Combining the advantages of both ant colony algorithm and double cluster heads, ADCAPTEEN is introduced in this paper. In ADCAPTEEN, one master cluster head (MCH) and one vice cluster head $(\mathrm{VCH})$ are chosen in each cluster to co-work on data collection, fusion, and transmission via the double cluster heads (DCH). MCH only communicates with $\mathrm{VCH}$. It avoids the direct communication between $\mathrm{MCH}$ and sink nodes. Simulation results show that ADCAPTEEN decreases the energy dissipation, improves node survival rate, and increases the network life cycle compared to APTEEN. However, there is only one intermediate node (IM_node), i.e., $\mathrm{VCH}$, is chosen per round in each cluster. Just one path is formed between $\mathrm{MCH}$ and sink node in each round. This paper introduces AMAPTEEN, combining the characteristics of the nature of ant colony algorithm to quickly identify optimal path [13] and APTEEN. It is an extension of ADCAPTEEN. The IM node is chosen, by using ant colony algorithm, as soon as there is data transmitted from $\mathrm{CH}$ to the sink node. It is chosen to avoid the direct communication between cluster head and sink nodes. A new optimal path is identified every time, but with likely different identified paths. Therefore, the scheme intends to assign tasks to the multiple paths for saving energy. Simulation results show that in the same network environment and parameter settings, AMAPTEEN further delays the time of node death, reduces the energy consumption of nodes, and improves the lifetime of network compared to ADCAPTEEN.

The rest of paper will fall into followings: Section 2, background briefs of previous work in energy efficiency using cluster-based routing; Section 3, the detailed description of the proposed algorithms of AMAPTEEN and ADCAPTEEN; Section 4, the simulation and analysis; and finally, Section 5, the conclusions.

\section{Related works}

\subsection{APTEEN protocol}

APTEEN protocol is developed based on LEACH with $\mathrm{CH}$ being selected in random. During the forming of clusters, it will generate random numbers ranging from 0 to 1 compared with a threshold, $T(n)$. The node is made as a $\mathrm{CH}$ for the current round, should the generated value $<T(n)$; otherwise, the node remains as a cluster member (CM) [3]. The threshold $T(n)$ can be expressed by Eq. (1).

$$
T(n)=\left(\begin{array}{cc}
\frac{p}{1-p^{*}\left(r \bmod \frac{1}{p}\right)} & \text { if } n \in G \\
0 \quad ; & \text { otherwise }
\end{array}\right.
$$

where $p$ is the elected probability of the MCHs among all the nodes, $r$ is the number of current round, and $G$ is the collections of the nodes that have not yet been selected as $\mathrm{MCH}$ nodes during the previous $\frac{1}{p}$ rounds.

Once $\mathrm{CH}$ is determined, it declares itself selected as the $\mathrm{CH}$ in this round and broadcasts the attribute, hard threshold (HT), soft threshold (ST), and count time (CT) parameters. Each node may acquire messages from one or more $\mathrm{MCH}$ and will choose its participation in the cluster which has the strongest received signal. After cluster formation, CM starts sensing continuously. When the value exceeds HT, sensed value (SV) is stored in an internal variable and the data is transmitted to $\mathrm{CH}$ according to TDMA schedule assigned for it. And then, $\mathrm{CM}$ node keeps on sensing. The sensed value will be 
stored and transmitted again only if the sensed value exceeds $\mathrm{HT}$, and meanwhile, the variations of sensed value in the transmitter and receiver exceed the ST [14]. In addition, as CT is surpassed, the sensed value does not exceed threshold value which causes no sensed data, the nodes are forced to transmit data to $\mathrm{CH}$. CT is the maximal time interval between two reports sent successively by a node. Since data transmission appears periodic, the sensed values of nodes are transmitted frequently to $\mathrm{CH}$. Reasonable selection on threshold values and CT can reduce the energy consumption.

\subsection{Sensor energy model}

Figure 1 demonstrates the radio energy model which is cast-off to evaluate the APTEEN as well as various APTEEN in this paper. For transmitting an $l$-bit message overd, the node energy consumption is given by

$$
\begin{aligned}
E_{T x}(l, d) & =E_{\text {elec }}(l)+E_{m p}(l, d) \\
& = \begin{cases}E_{\text {elec }} * l+l * \varepsilon_{f s} * d^{2} ; & d<d_{0} \\
E_{\text {elec }} * l+l * \varepsilon_{m p} * d^{4} ; & d \geq d_{0}\end{cases}
\end{aligned}
$$

and the energy consumption for receiving the $l$-bit message over $d$ is given by

$$
E_{R x}(l)=l * E_{\text {elec }}
$$

In the above equation, $E_{\text {elec }}$ denotes the transmitting or receiving circuit loss, $d_{0}$ denotes the criticality value $d_{0}=\frac{\sqrt{\varepsilon_{s}}}{\sqrt{\varepsilon_{m p}}}$. If the communication distancedis larger than the threshold distance $d_{0}$, i.e., $d \geq d_{0}$, the multipath fading channel mode is used $\left(d^{4}\right.$ consumption loss). The coefficients for power amplification is denoted by $\varepsilon_{m p}$; otherwise, the free space channel model is chosen ( $d^{2}$ consumption loss), and the coefficients for power amplification is denoted by $\varepsilon_{f s}$.

\subsection{DCH protocol}

The DCH routing protocol for the WSN is that the nodes are grouped into clusters and the routing algorithm allows to selecting a $\mathrm{MCH}$ and a $\mathrm{VCH}$ in each cluster. The two kinds of cluster heads play different roles in the network. They cooperate mutually to complete the collection and aggregation of primary data and transmission from cluster to the sink node. Nodes in the cluster transmit data to $\mathrm{MCH}$ which collects and fuses data and sends aggregation data to the same cluster of $\mathrm{VCH}$. VCH transmits data to far sink nodes. Since the direct communication is avoided between $\mathrm{MCH}$ and sink node, energy consumption is reduced to a large degree and the lifetime of the whole network is extended.

\section{Propose protocols}

In this section, the improved algorithms based on ant colony will be explained in detail, so will their characteristics.

\subsection{ADCAPTEEN protocol}

In this paper, Fig. 2 presents the ADCAPTEEN network model. The structure of the network model consists of $\mathrm{CM}, \mathrm{MCH}, \mathrm{VCH}$, and sink node in four parts. In each cluster, a $\mathrm{MCH}$ and a $\mathrm{VCH}$ are selected. The $\mathrm{CM}$ is responsible for collecting and transmitting data to the $\mathrm{MCH}$. The $\mathrm{MCH}$ collects and merges the data and sends the converged data to the $\mathrm{VCH}$ of the cluster, and the $\mathrm{VCH}$ forwards the data to the sink node at a far distance.

It is periodic for this protocol to implement its process. Each round is divided into two phases: the phase of cluster formulation and intra-cluster data transmission and the phase of $\mathrm{VCH}$ selection and stable state. Firstly, it optimizes the computing method of the threshold, $T(n)$. The improved $T(n)$ considers the residual energy of nodes. The improved threshold $T(n)$ can be expressed with Eq. (4). The $\mathrm{MCH}$ is selected by considering the remaining energy of each node. The choice of $\mathrm{MCH}$ is more reasonable.

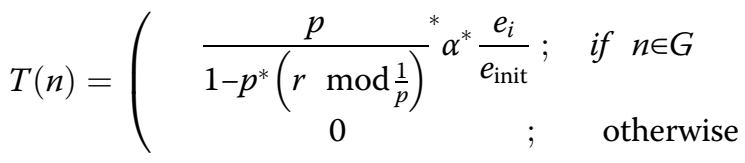

where $e_{i}$ is the current remaining energy $i$ of node, $e_{\text {init }}$ is initial energy $i$ of node, and $\alpha$ is the regulatory factor.

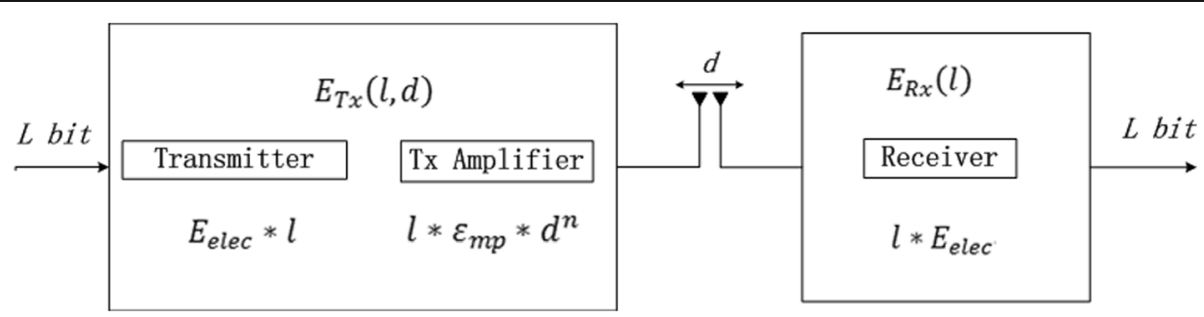

Fig. 1 Communication energy consumption model of WSN. This figure demonstrates the radio energy model which is cast-off to evaluate the APTEEN as well as various APTEEN in this paper 


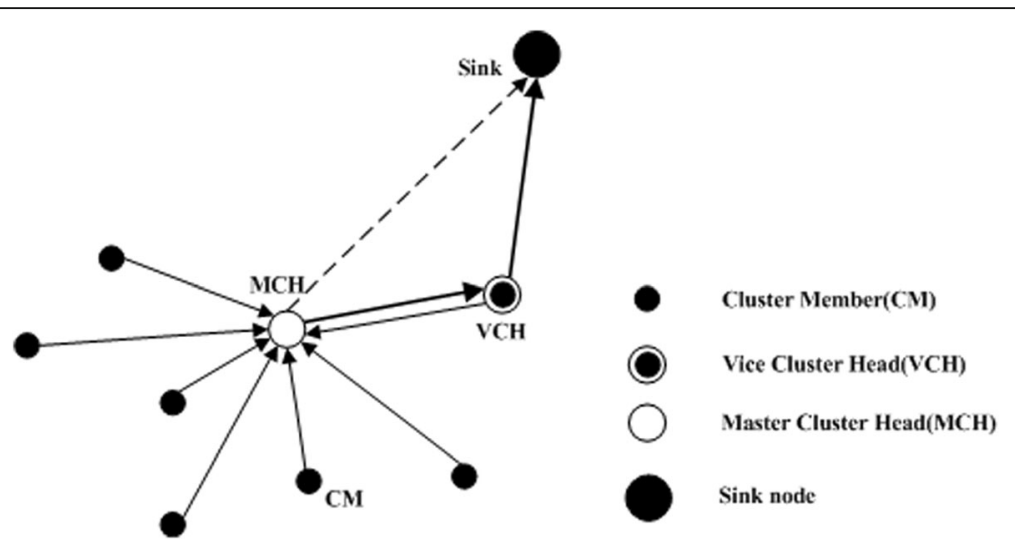

Fig. 2 ADCAPTEEN network model. The ADCAPTEEN network model consists of $C M, M C H, V C H$, and sink node in four parts. In each cluster, a $\mathrm{MCH}$ and a VCH are selected. The CM is responsible for collecting and transmitting data to the $\mathrm{MCH}$. The MCH collects and merges the data, sends the converged data to the VCH of the cluster, and the VCH forwards the data to the sink node at a far distance

\subsubsection{Cluster formulation and intra-cluster data transmission}

Each node compares the generated random number with Eq. (4) threshold to determine the current round of the $\mathrm{MCH}$. If it is $\mathrm{MCH}$, broadcast news announce them as the current round of $\mathrm{MCH}$.

The non- $\mathrm{MCH}$ node receives the broadcast information, determines which cluster to join according to the received signal strength, and sends a join request to the $\mathrm{MCH}$ with the strongest received signal to become the cluster CM; the nodes are divided dynamically into several clusters and establish a virtual link between the $\mathrm{MCH}$ and the $\mathrm{CMs}$ by the ACO algorithm (initial pheromone concentration value is 0 ).

After the $\mathrm{CM}$ receives the message, the $\mathrm{MCH}$ assigns TDMA time slots to all the CMs in the cluster and sends them to the CM. In the phase of cluster formulation, node energy in the network has been consumed of a different degree. The pheromone concentration is then calculated according to Eq. (5), which is expressed as:

$$
\tau_{m i}(t)=\frac{Q^{*}}{d_{m i}}(1-\rho)
$$

Pheromone volatilization rate is defined as $\rho, \rho$ $=\frac{E_{\text {init }}-E_{m}}{E_{\text {init }}}$, and it is related to node energy sending messages, where $E_{\text {init }}$ is initial energy of $\mathrm{MCH}$ node, and $E_{m}$ is current energy of $\mathrm{MCH}$ node. $d_{m i}$ is distance from node $i$ to $\mathrm{MCH}$. $Q$ is a preset parameter; the value of $Q$ is 10 in this paper.

All CMs send data to MCHs when the conditions are met for data transmission of the proposed algorithm from $\mathrm{CM}$ to $\mathrm{MCH}$. The conditions are as same as APTEEN, i.e. , including ST, HT, and CT comparison. $\mathrm{MCH}$ send routing package, let CM report its energy message. CM send routing package carrying the current remaining energy information. $\mathrm{MCH}$ calculate the increase in pheromone concentration according to CM's energy. The increased amount of pheromone in the corresponding link can be expressed as:

$$
\Delta \tau_{m i}=\beta^{*} \frac{e_{i}}{e_{\text {init }}}
$$

where $e_{i}$ is the current energy $i$ of node, $e_{\text {init }}$ is the initial energy $i$ of node, and $\beta$ is the regulatory factor.

\subsubsection{VCH selection and stable state}

Then, according to Eqs. (5) and (6), the pheromone concentration update expression is concluded as follows:

$$
\tau_{m i}=\tau_{m i}(t)+\Delta \tau_{m i}=\frac{Q^{*}}{d_{m i}} \frac{E_{m}}{E_{\text {init }}}+\beta^{*} \frac{e_{i}}{e_{\text {init }}}
$$

According to Eq. (7), the pheromone concentration on the link between the $\mathrm{MCH}$ and $\mathrm{CMs}$ can be obtained. $\mathrm{MCH}$ selects the $\mathrm{CM}$ node with the largest pheromone concentration on the link as $\mathrm{VCH}$. $\mathrm{VCH}$ is selected to require the $\mathrm{CM}$ to be as close to the $\mathrm{MCH}$ as possible, at the same time, the remaining energy of $\mathrm{CM}$ as high as possible.

$\mathrm{CM}$ sends the sensed data to $\mathrm{MCH}$ in its allocated TDMA slot. When $\mathrm{MCH}$ receives the data from all the CMs, it collects and aggregates the data and sends the merging message to $\mathrm{VCH}$ which receives and transmits the merging message to far sink node. The protocol requires only communication between intra-cluster nodes and no communication between clusters.

Once VCH is selected, it will not change until it enters the next round. When entering the next round, pheromone concentration between nodes will be cleared.

Figure 3 shows the allocation process of ADCAPTEEN. Obviously, owing to node communication only between $\mathrm{MCH}$ and $\mathrm{VCH}, \mathrm{VCH}$ performs most of energy consumption. $\mathrm{MCH}$ has no direct communication with sink node, so it balances energy consumption, improves 


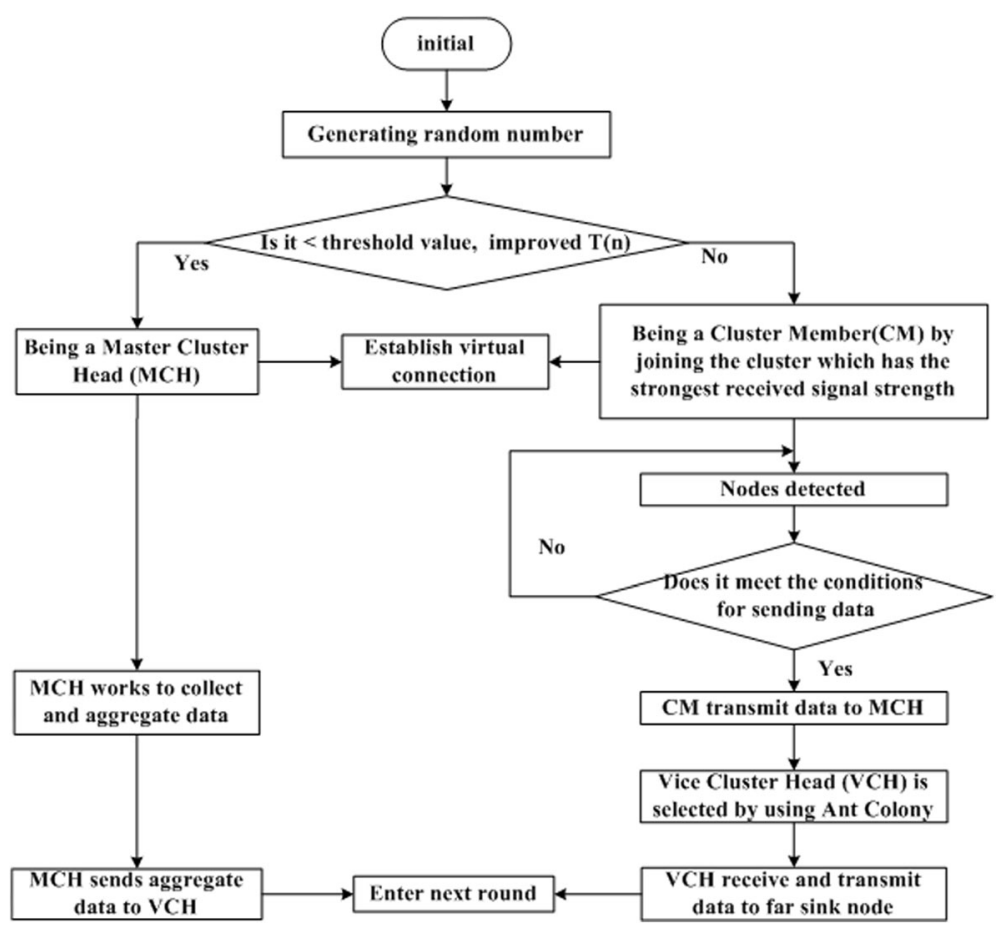

Fig. 3 Flow of the ADCAPTEEN protocol. The generated random number is compared with the formula threshold to determine MCH. The non-MCH node sends a join request to the $\mathrm{MCH}$ with the strongest received signal to become the cluster $\mathrm{CM}$. Nodes are divided dynamically into several clusters. A virtual link between the $\mathrm{MCH}$ and the $\mathrm{CMs}$ is established. $\mathrm{MCH}$ selects the $\mathrm{CM}$ node with the largest pheromone concentration on the link as VCH. Once VCH is selected, it will not change until it enters the next round. CM sends the sensed data to MCH in its allocated TDMA slot. When MCH receives the data from all the CMs, it collects and aggregates data and sends the merging message to VCH which receives and transmits the merging message to far sink node

the number of surviving nodes, and prolongs the life cycle of the WSN.

\subsection{AMAPTEEN protocol}

This section focuses on the AMAPTEEN. In the pheromone update formulas, AMAPTEEN considers the distances between $\mathrm{CM}$ and $\mathrm{CH}$ node and the residual energy of CM. The AMAPTEEN protocol is illustrated, and its characteristics are explained here. Its network model is as below in Fig. 4. The structure of the network model mainly consists of $\mathrm{CM}, \mathrm{CH}$, IM_node, and sink node four parts. $\mathrm{CM}$ sends the sensed data to $\mathrm{CH}, \mathrm{CH}$ collects and fuses the data, and sends the fusion of the data to IM node. IM_node forwards the data to a distant sink node.

In this paper, the AMAPTEEN protocol's implementation procedure appears periodic. Each round is divided into two phases: the phase of cluster formulation and intra-cluster data transmission and the phase of optimal path selection and inter-cluster data transmission.

\subsubsection{Cluster formulation and intra-cluster data transmission}

Each node compares the generated random number with Eq. (4) threshold to determine the current round of the

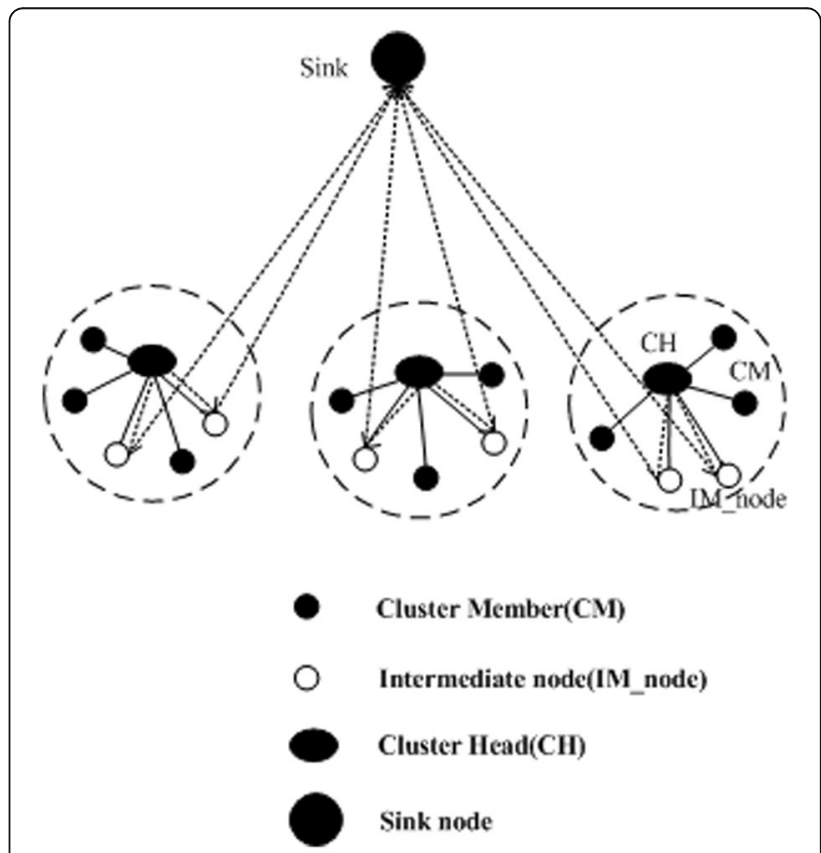

Fig. 4 AMAPTEEN network model. The AMAPTEEN network model consists of $\mathrm{CM}, \mathrm{CH}$, IM_node, and sink node four parts. CM sends the sensed data to $\mathrm{CH}$, and $\mathrm{CH}$ collects and fuses data and sends the fusion of data to IM_node. IM_node forwards the data to a distant sink node 
$\mathrm{CH}$. Outside, the phase of cluster formulation and intracluster data transmission is the same as ADCAPTEEN. The nodes are divided dynamically into several clusters.

\subsubsection{Optimal path selection and inter-cluster data transmission}

The optimal path selection phase of the AMAPTEEN protocol replaced $\mathrm{VCH}$ selection of the ADCAPTEEN protocol. In this state, using ant colony algorithm, $\mathrm{CH}$ selects the $\mathrm{CM}$ node with the largest pheromone concentration on the link according to Eq. (7) as IM_node.

$\mathrm{CM}$ sends the sensed data to $\mathrm{CH}$ in its allocated TDMA slot. When $\mathrm{CH}$ receives the data from all the CMs, it collects and aggregates data and sends the merging message to IM_node. The IM_node forwards the message to far sink node. The protocol requires only communication between intra-cluster nodes, and no communication between clusters.

The IM_node is chosen by $\mathrm{CH}$ whenever there are data transmitted from $\mathrm{CH}$ to sink node. When entering the next round, pheromone concentration will be cleared between nodes.

The flow of the AMAPTEEN protocol is depicted in Fig. 5.

In AMAPTEEN protocol, the residual energy of the node is considered in the choice of cluster head. The choice of cluster head is more reasonable. The IM_node is chosen as soon as there is data transmitted from $\mathrm{CH}$ to the sink node. The choice of IM_node depends on node maximal pheromone concentration, and thus, choosing the data transmission path is relatively optimal. And it is likely to be different, so there will be formed multiple data transfer paths from $\mathrm{CH}$ to sink. Streaming data to multiple paths reduces energy consumption, improves node survival rate, and extends life cycle of network further compared with ADCAPTEEN.

\section{Simulation and analysis}

The simulation model embraces many sensor nodes grouped into clusters and one fixed sink node. The OPNET is used to evaluate ADCAPTEEN compared with APTEEN and AMAPTEEN compared with ADCAPTEEN. In the experiment, 50-node network and 100-node network are randomly distributed between the area of $(x=0, y=0)$ and $(x=200, y=125)$ with the sink node at $(x=75, y=175)$. The network models of WSN are shown in Figs. 6 and 7. It is periodic for this protocol to implement its process. Each round time is $20 \mathrm{~s}$.

The simulation parameters are shown in Table 1. CM senses data continuously and assumes the detection times (DT) are $K$ in per round, then CMs are forced to transmit data to $\mathrm{MCH}$ or $\mathrm{CH}$ if the number of times which $\mathrm{CM}$ is not transmitting data to $\mathrm{CH}$ exceeds $\mathrm{CT}$.

\subsection{APTEEN simulation and analysis}

Network life cycle of the algorithms is closely related with the probability of elected cluster heads. After modeling and simulation in OPNET, network life cycle comparison with probabilities are $0.15,0.2$ and 0.25 respectively is shown as Fig. 8. The horizontal axis is the simulation time, the vertical shaft is the number of live nodes. As can be seen from Fig. 8, the smaller $p$ values selected, the longer network life cycle. Therefore, the probability of elected cluster head elected cluster $p$ is very important to improve the network performance of APTEEN protocol. For the network constructed in this paper, $p=0.15, p=0.2$, and $p=0.25$ belong to a reasonable range. $p$ takes a value of 0.2 in APTEEN and related protocols in this paper.

To verify the validity and scalability of the network, we build 100 nodes network topology. The network model is shown in Fig. 7. Figure 9 shows the network life cycle of APTEEN compared 100 nodes with 50-node network topology. The network life cycles of both topologies show a downward trend stably. And the death time of the first node is also very close, so this paper established APTEEN network topology effectively. Randomly chosen as one of the nodes, Fig. 10 is a single-node energy consumption of APTEEN in different network scales. The horizontal axis is the simulation time, and vertical shaft is the node energy (J). From Fig. 10 , we can see that increasing the number of nodes makes node energy consumption more stable. The description of the network has strong scalability, suitable for large-scale network.

\subsection{Comparison of APTEEN and ADCAPTEEN}

In creating a new ADCAPTEEN scene, ADCAPTEEN routing protocol is compared with APTEEN routing protocol in OPNET. Figure 11 shows the network life cycle of ADCAPTEEN compared 100 nodes with the 50 nodes network topology. We can see that established ADCAPTEEN network topology is also effective. Compared with Fig. 9, ADCAPTEEN protocol results in a greater network life cycle than the APTEEN protocol under two network topologies. ADCAPTEEN outperforms APTEEN in terms of scalability. It is suitable for large-scale network.

The comparison of life cycle under APTEEN and ADCAPTEEN routing protocol is shown in Fig. 12. Figure 12 shows that about after $450 \mathrm{~s}$, APTEEN begin node dies. In about $3600 \mathrm{~s}$, most nodes run out of energy, with only a few nodes surviving. ADCAPTEEN begin node dies in about $500 \mathrm{~s}$, and about after $3600 \mathrm{~s}$, ADCAPTEEN has many nodes alive. Compared with APTEEN, ADCAPTEEN routing protocol delays the time of node death, increases the node survival rate, and extends the network survival time. 


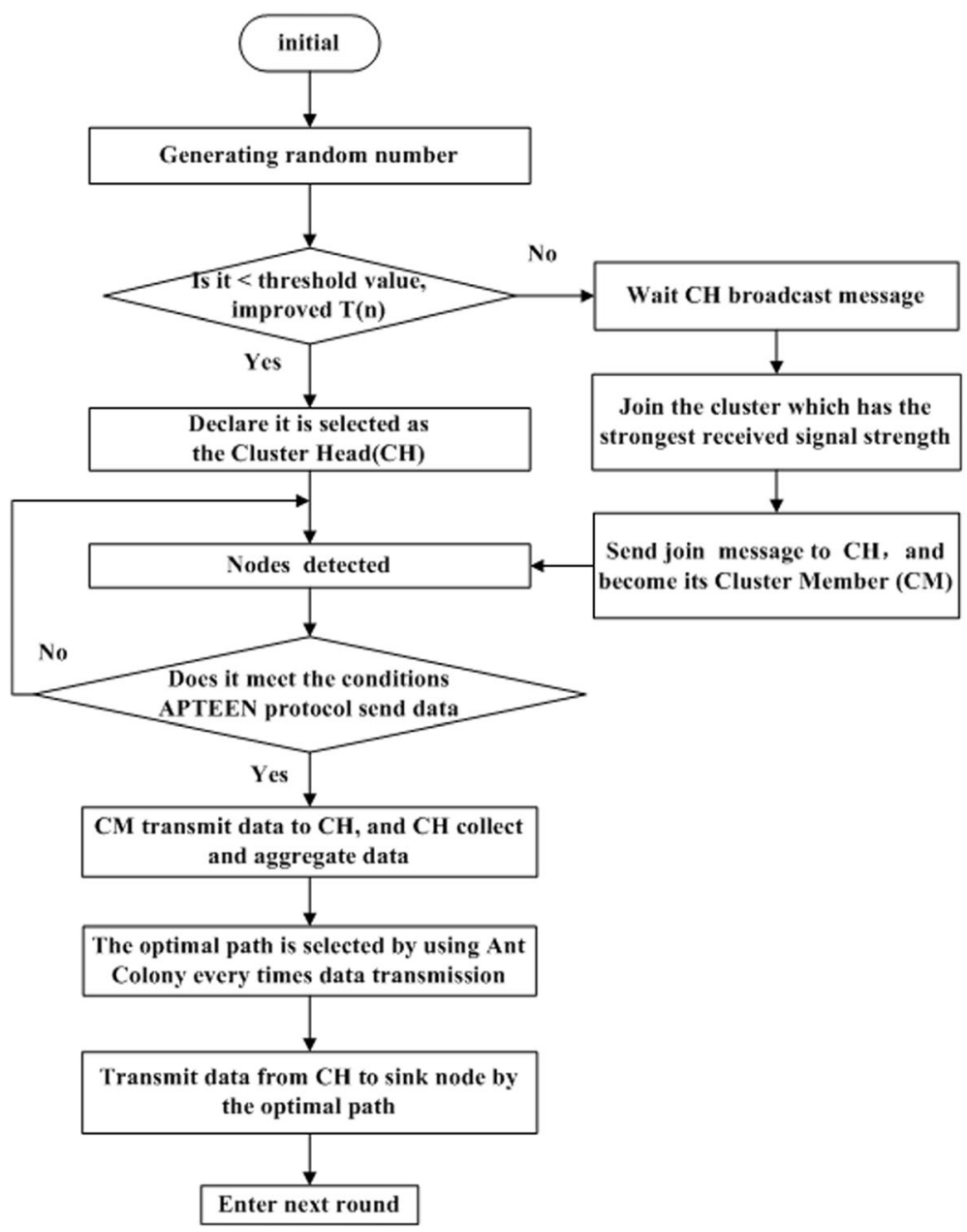

Fig. 5 Flow of the AMAPTEEN protocol. The generated random number is compared with the formula threshold to determine $\mathrm{CH}$. The non- $\mathrm{CH}$ node selects the $\mathrm{CH}$ with the strongest received signal to join and become the cluster's $\mathrm{CM}$. Nodes are divided dynamically into several clusters. A virtual link between the $\mathrm{CH}$ and the $\mathrm{CMs}$ is established. $\mathrm{CH}$ selects the $\mathrm{CM}$ node with the largest pheromone concentration on the link as IM_node. The IM_node is chosen by $\mathrm{CH}$ whenever there are data transmitted from $\mathrm{CH}$ to sink node. CM sends the sensed data to $\mathrm{CH}$ in its allocated TDMA slot. When $\mathrm{CH}$ receives the data from all the $\mathrm{CMs}$, it collects and aggregates data and sends the merging message to IM_node. The IM_node forwards the message to far sink node. The choice of IM_node depends on node maximal pheromone concentration, and thus, choosing the data transmission path is relatively optimal. And it is likely to be different, so there will be formed multiple data transfer paths from $\mathrm{CH}$ to sink

The energy consumption under APTEEN and ADCAPTEEN routing protocol is shown in Fig. 13. It shows ADCAPTEEN routing protocol reduced the energy consumption of a single node compared with APTEEN.

\subsection{Comparison of ADCAPTEEN and AMAPTEEN}

Creating a new AMAPTEEN scene, AMAPTEEN routing protocol is compared with ADCAPTEEN routing protocol in OPNET.

The network life cycle of AMAPTEEN compared 100 nodes with the 50-node network topology is shown in Fig. 14. Compared with Figs. 9 and 11, it can be seen that AMAPTEEN also has good reliability and scalability and fits the large-scale network.

Figure 15 shows the comparison of network lifetime under ADCAPTEEN and AMAPTEEN. We can see that AMAPTEEN has further extended the network life cycle and improved the number of surviving nodes compared with ADCAPTEEN.

The energy consumption of single node compares ADCAPTEEN and AMAPTEEN is shown in Fig. 16. The results show that the energy consumption of AMAPTEEN routing algorithm is smaller than ADCAPTEEN. AMAPTEEN chooses IM_node as soon as there was data transmission from $\mathrm{CH}$ and sink node. Streaming data to multiple paths, the energy consumption is quiet reduced. 


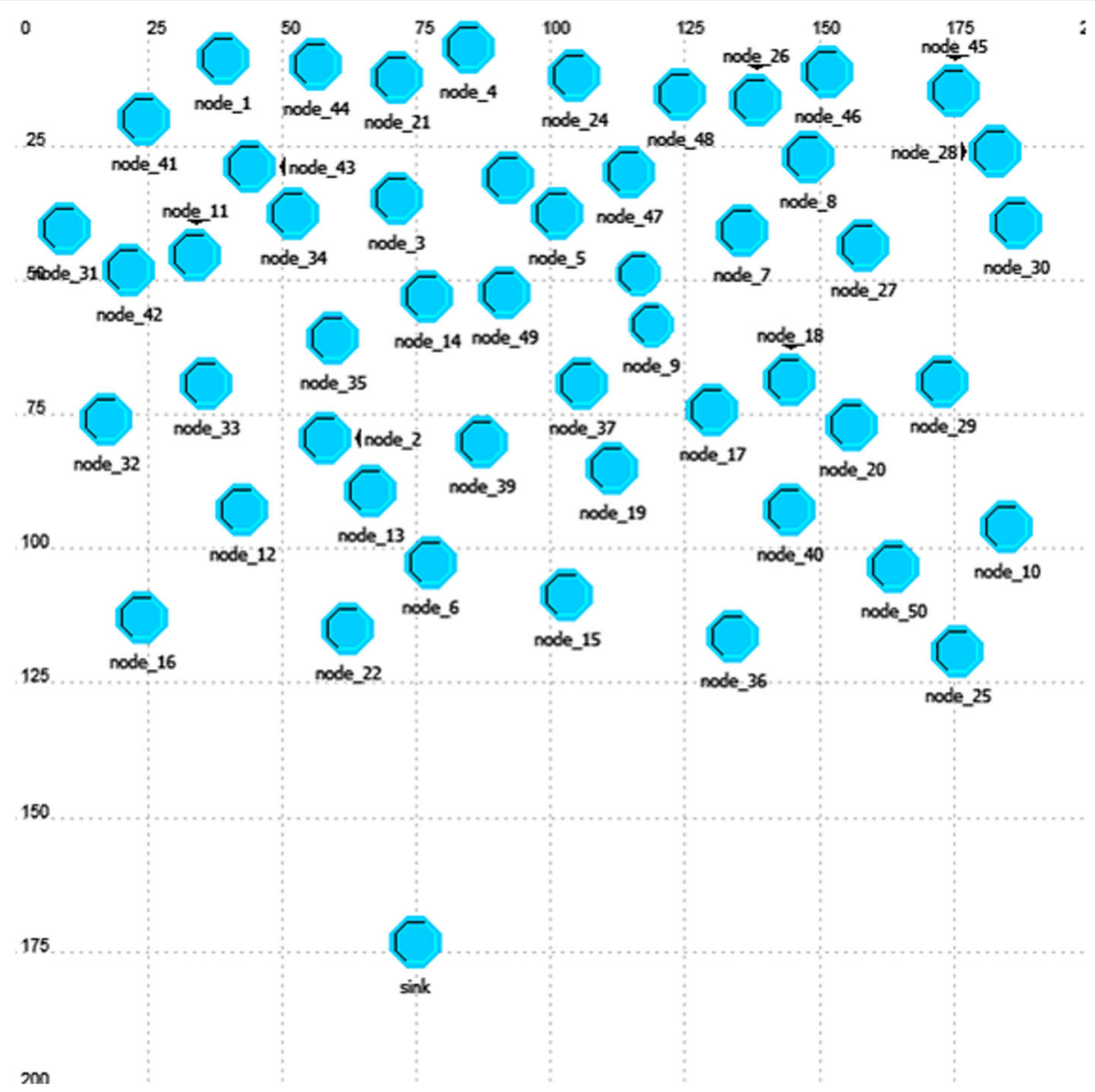

Fig. 6 The 50-node network model of the WSN. The 50-node network model of WSN is shown in this figure. Fifty nodes are randomly distributed between the area of $(x=0, y=0)$ and $(x=200, y=125)$ with the sink node at $(x=75, y=175)$

\section{Conclusions}

This paper presents a double cluster heads Adaptive Threshold-sensitive Energy Efficient Network based on ant colony (ADCAPTEEN) and a Multiple Adaptive Threshold-sensitive Energy Efficient Network based on ant colony (AMAPTEEN) in wireless sensor networks (WSN). Two proposed protocols modify traditional $T(n)$, and $\mathrm{MCH}$ and $\mathrm{CH}$ are selected by considering the residual energy of nodes. Making the choice of $\mathrm{MCH}$ and $\mathrm{CH}$ is more reasonable. This paper presents ADCAPTEEN to choose one $\mathrm{VCH}$ by $\mathrm{MCH}$ per round in each cluster, selected $\mathrm{VCH}$ to avoid the $\mathrm{MCH}$ and sink nodes communicate directly. It reduces the energy dissipation and achieves the improvement on network life cycle. Considering ADCAPTEEN will cause imbalanced energy consumption due to one path completing multiple data transmission between $\mathrm{MCH}$ and sink node in each round. IM_node will be chosen if there was data transmission each time in AMAPTEEN from $\mathrm{CH}$ and sink node. Forming multipath using ant colony, AMAPTEEN reduces energy consumption, improves node survival rate, and extends life cycle of network further compared with ADCAPTEEN.

\section{Results and discussion \\ 6.1 ADCAPTEEN}

Compared with APTEEN, ADCAPTEEN reduces energy dissipation, improves node survival rate, and extends network life cycle. The proposed algorithm has good scalability, and it is suitable for large-scale network. Because ADCAPTEEN optimizes the cluster head election method compared with APTEEN. And one master cluster head $(\mathrm{MCH})$ and one vice cluster head ( $\mathrm{VCH})$ will be selected in each cluster. The double cluster heads (DCH) can co-work on data collection, fusion, transition, etc. $\mathrm{MCH}$ has no direct communication with sink node, and $\mathrm{VCH}$ performs most of energy consumption. 


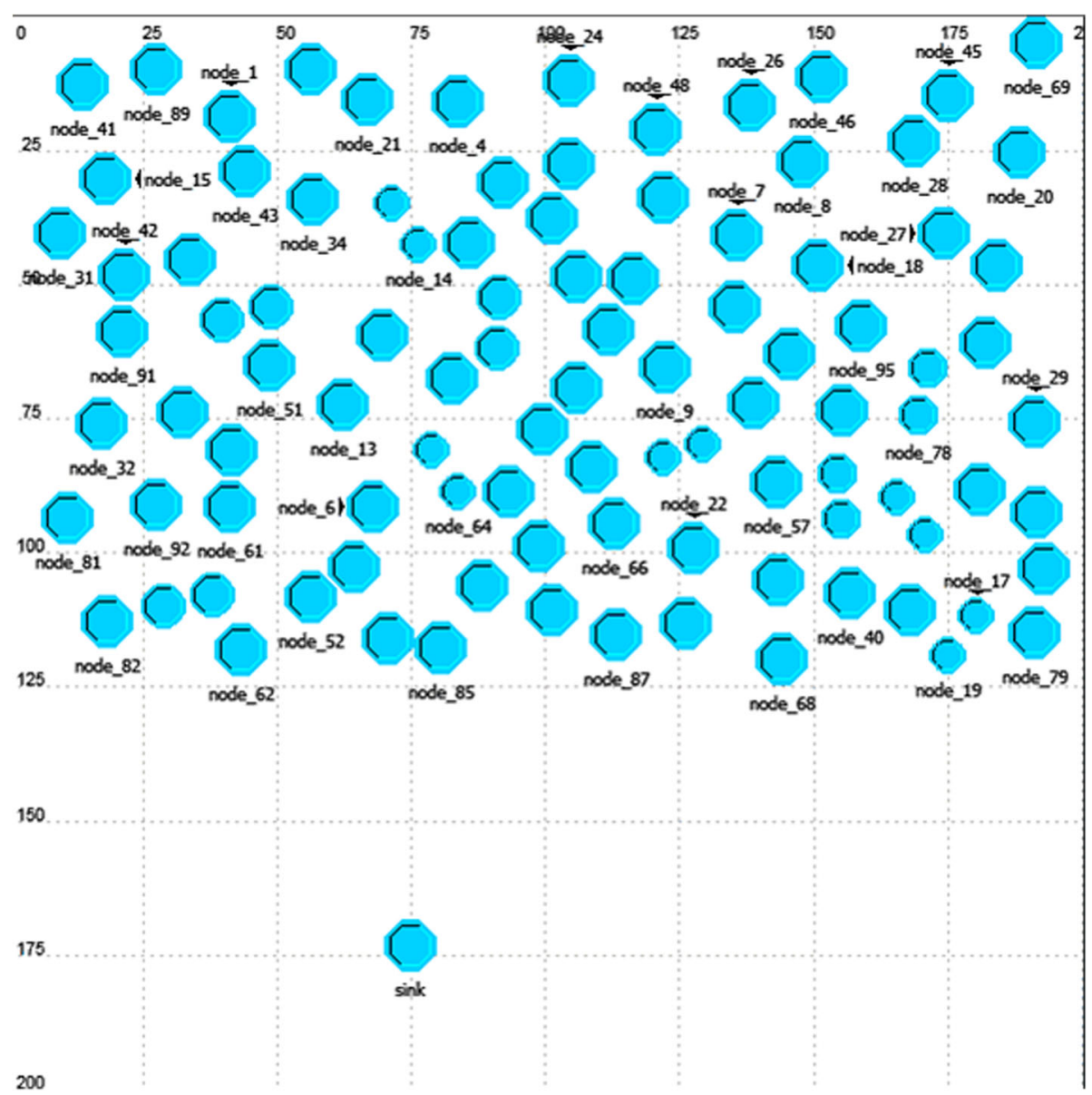

Fig. 7 The 100-node network model of the WSN. The 100-node network model of WSN is shown in this figure. One hundred nodes are randomly distributed between the area of $(x=0, y=0)$ and $(x=200, y=125)$ with the sink node at $(x=75, y=175)$

\subsection{AMAPTEEN}

AMAPTEEN delays the time of node death, balances energy consumption, and extends network lifetime further operating in the same settings compared with ADCAPTEEN. The proposed algorithm also has good scalability, and it is suitable for large-scale network.

Table 1 Parameters

\begin{tabular}{llll}
\hline Parameter & Value & Parameter & Value \\
\hline Initial energy & $0.5 \mathrm{~J}$ & $H T$ & 5 \\
Data packet & 4100 bytes & $S T$ & 0.1 \\
Control packet & 100 bytes & $C T$ & 3 \\
$P$ & 0.2 & $K$ & 10 \\
$a$ & 1 & $\beta$ & 10 \\
$E_{\text {elec }}$ & $50 \mathrm{~nJ} / \mathrm{bit}$ & $\varepsilon_{f s}$ & $100 \mathrm{pJ} / \mathrm{bit} / \mathrm{m}^{2}$ \\
$E_{D A}$ & $5 \mathrm{~nJ} / \mathrm{bit} / \mathrm{signal}$ & $\varepsilon_{m p}$ & $0.0013 \mathrm{p} / \mathrm{bit} / \mathrm{m}^{4}$ \\
\hline
\end{tabular}

This paper evaluates the network performance index that mainly includes the network life cycle, the node survival rate, and the node energy consumption. A network life cycle is an important index to evaluate the performance of the clustering routing protocol. It refers to the time when the first node runs out of energy in WSN
This is because AMAPTEEN is the optimization of ADCAPTEEN. And $\mathrm{CH}$ selects intermediate node (IM_node) multiple times with ant colony algorithm per round in each cluster and this way forms multiple routes to transmit data.

\section{Methods \\ 7.1 ADCAPTEEN}

ADCAPTEEN improved threshold $T(n)$, and the improved $T(n)$ considers the residual energy of nodes. $\mathrm{MCH}$ is selected by comparing the generated random number with the improved threshold $T(n)$. The nodes are divided dynamically into several clusters. In each cluster, to establish a virtual link between $\mathrm{MCH}$ and $\mathrm{CM}$ by the $\mathrm{ACO}$ algorithm. $\mathrm{MCH}$ selects the $\mathrm{CM}$ node with the largest pheromone concentration on the link according to using ant colony algorithm as $\mathrm{VCH}$. Once $\mathrm{VCH}$ is selected, it will not change until it enters the next round. $\mathrm{CM}$ is responsible for collecting and transmitting data to the $\mathrm{MCH}$. The $\mathrm{MCH}$ collects and merges the data, sends the converged 


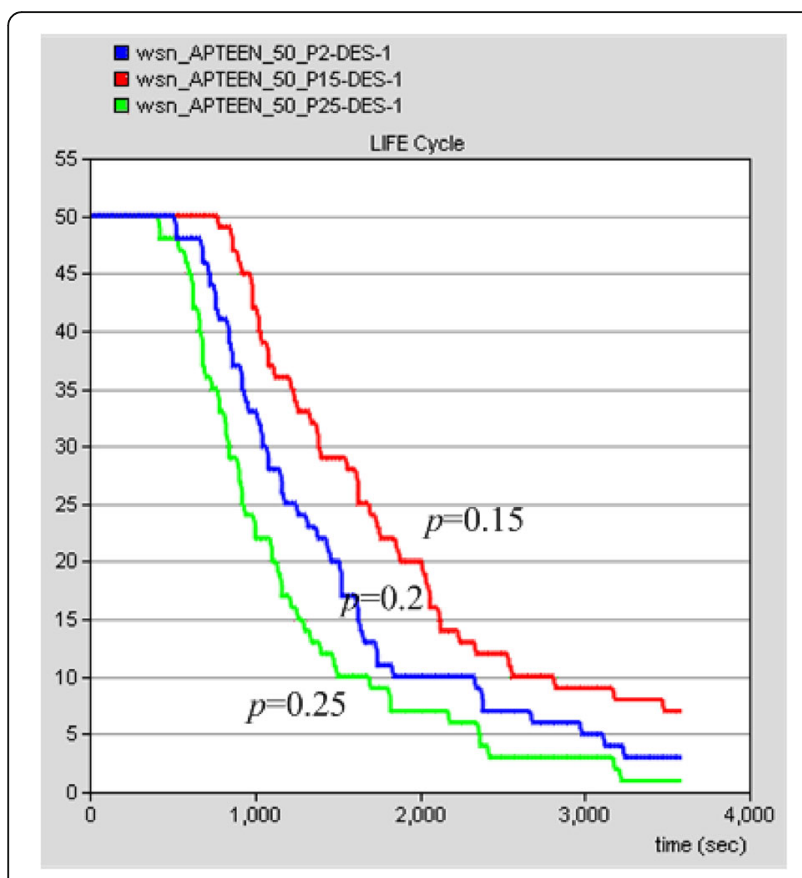

Fig. 8 The life cycle of APTEEN in different probability. Network life cycle comparison with probabilities is $0.15,0.2$, and 0.25 respectively in the 50-node network scene. As can be seen from the figure, the smaller $p$ values selected, the longer network life cycle

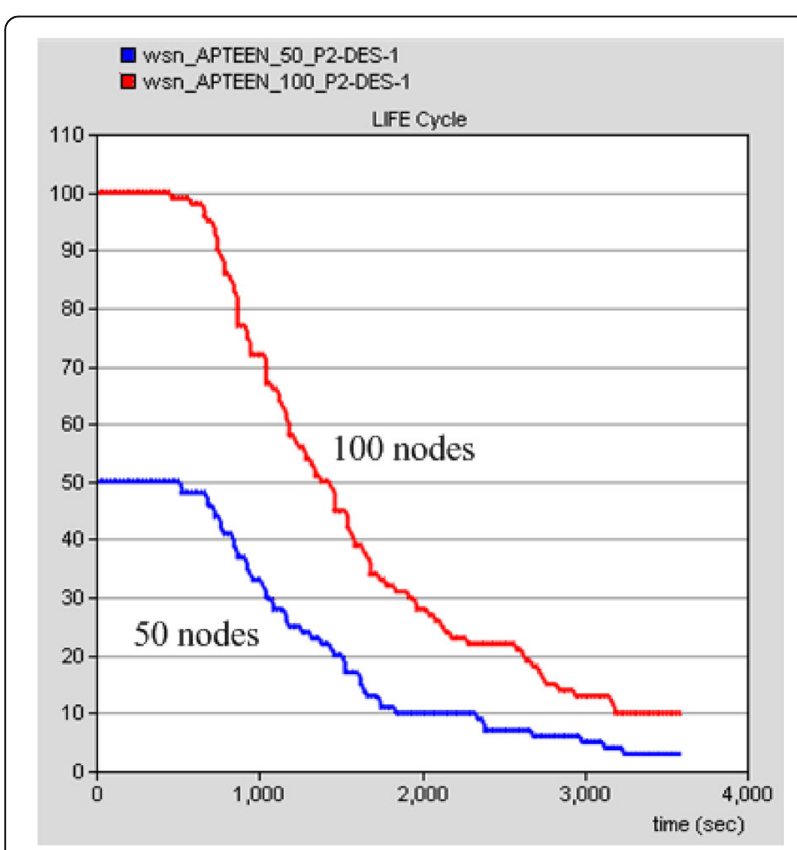

Fig. 9 The life cycle of APTEEN compared in different scales. This figure shows the network life cycle of APTEEN compared 100 nodes with 50-node network topology. The network life cycles of both topologies show a downward trend stably. And the death time of the first node is also very close, so this paper established APTEEN network topology effectively

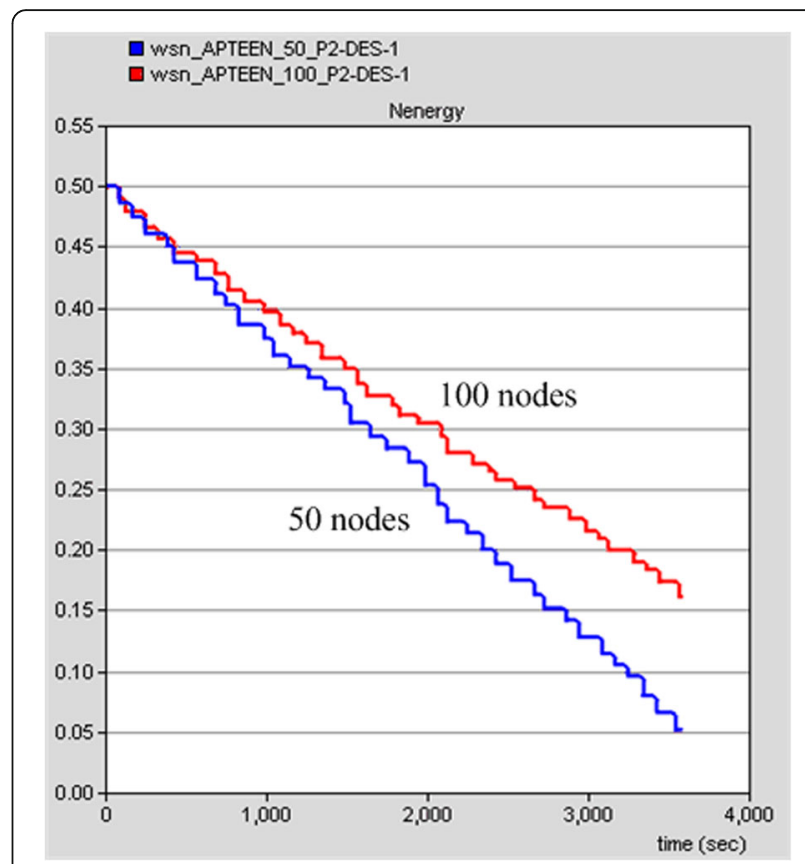

Fig. 10 Energy consumption of APTEEN compared in different scales. This figure shows a single-node energy consumption of APTEEN in different network scales. From this figure, we can see that increasing the number of nodes makes the node energy consumption more stable

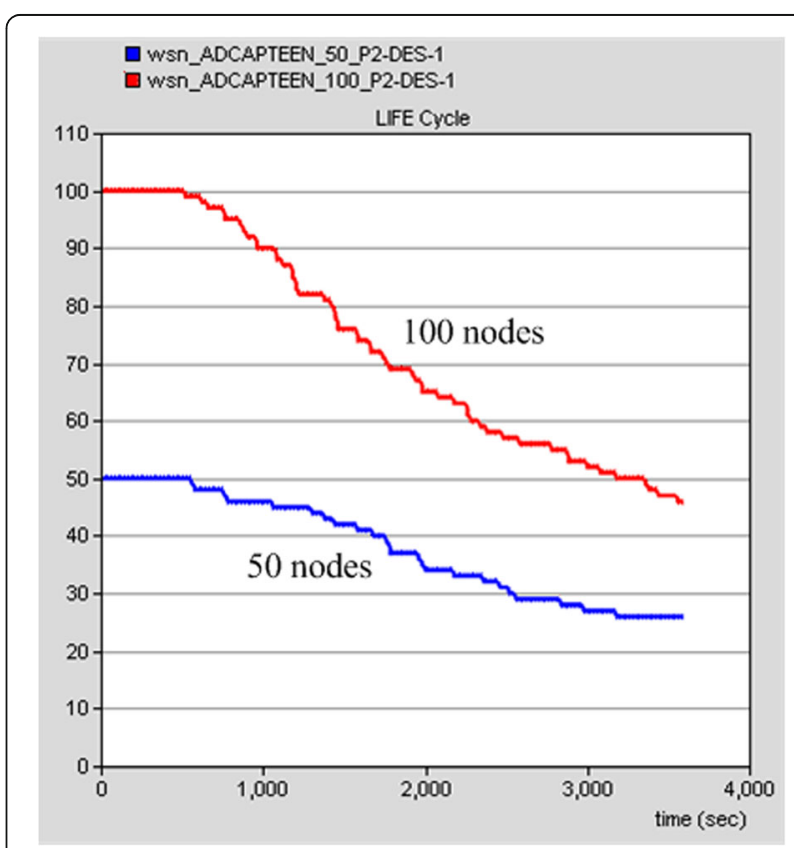

Fig. 11 The life cycle comparison of ADCAPTEEN in different scales. This figure shows the network life cycle of ADCAPTEEN compared 100 nodes with the 50-node network topology. We can see that established ADCAPTEEN network topology is also effective. Compared with Fig. 9, ADCAPTEEN protocol results in a greater network life cycle than the APTEEN protocol under two network topologies. ADCAPTEEN outperforms APTEEN in terms of scalability. It is suitable for large-scale network 


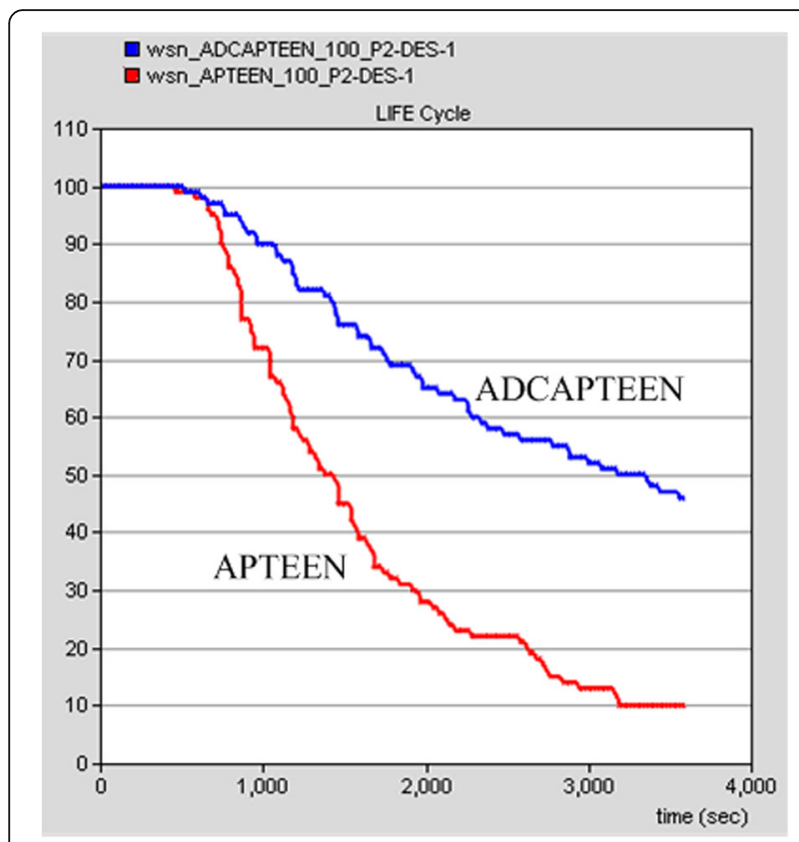

Fig. 12 Comparison of a life cycle between APTEEN and ADCAPTEEN. The comparison of a life cycle under APTEEN and ADCAPTEEN routing protocol is shown in this figure. This figure shows that about after $450 \mathrm{~s}$, APTEEN begin node dies. In about $3600 \mathrm{~s}$, most nodes run out of energy, with only a few nodes surviving. ADCAPTEEN begin node dies in about $500 \mathrm{~s}$, and about after $3600 \mathrm{~s}$, ADCAPTEEN have many nodes alive. Compared with APTEEN, ADCAPTEEN routing protocol delays the time of node death, increases the node survival rate, and extends the network survival time

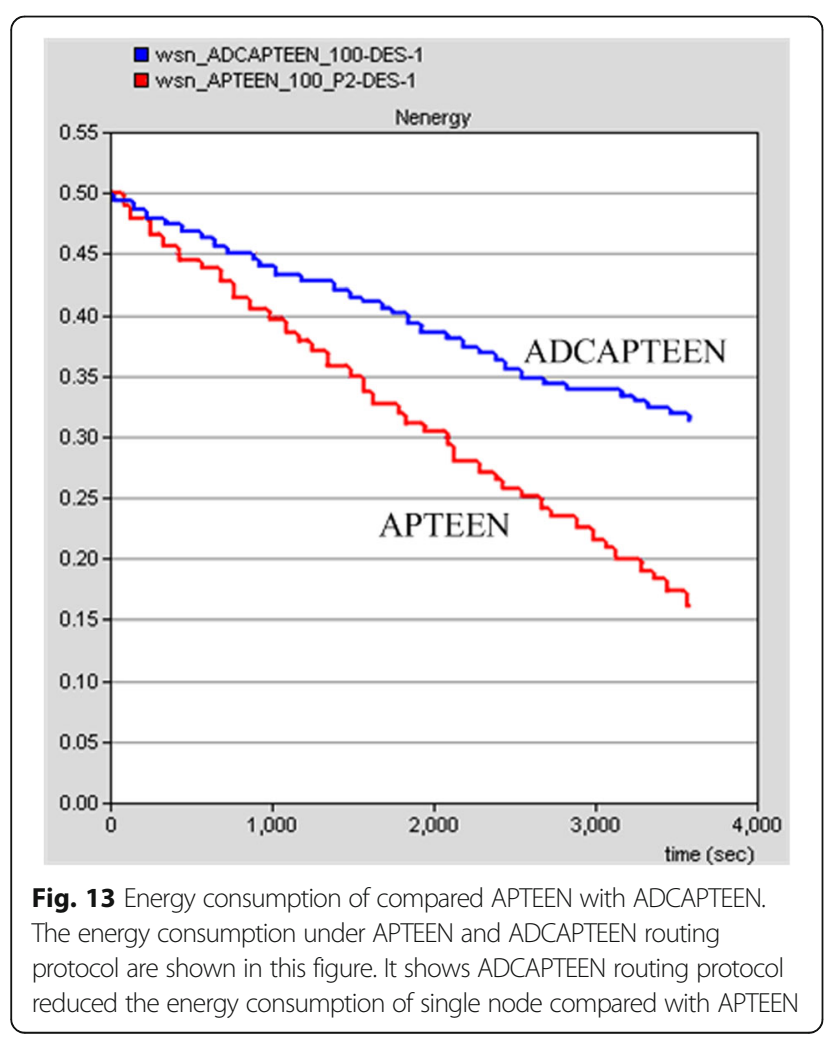

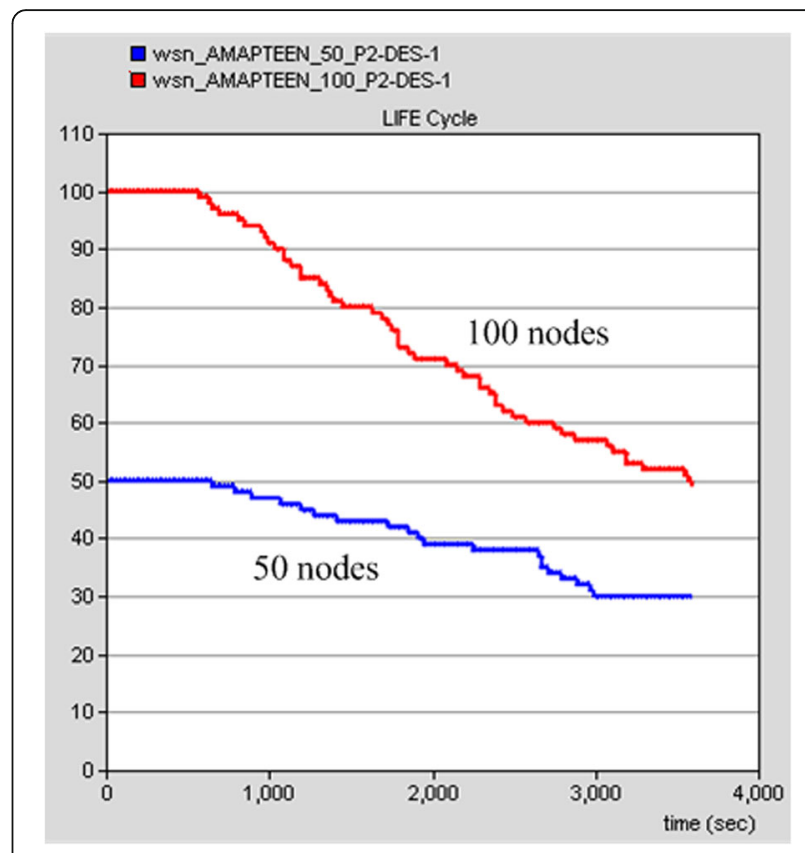

Fig. 14 The life cycle comparison of AMAPTEEN in different scales. The network life cycle of AMAPTEEN compared 100 nodes with the 50-node network topology is shown in this figure. Compared with Fig. 9 and Fig. 11, it can be seen that AMAPTEEN also has good reliability and scalability and fits the large-scale network

data to the $\mathrm{VCH}$ of the cluster, and the $\mathrm{VCH}$ forwards the data to the sink node at a far distance. $\mathrm{MCH}$ has no direct communication with sink node, and $\mathrm{VCH}$ performs most of energy consumption. Therefore, ADCAPTEEN reduces energy consumption, improves the number of surviving nodes, and prolongs the life cycle of the WSN compared with APTEEN.

\subsection{AMAPTEEN}

AMAPTEEN is the optimization of ADCAPTEEN to make routes more stable and energy-efficient. The generated random number is compared with the improved threshold $T(n)$ to determine the current round of the $\mathrm{CH}$. Using the ant colony algorithm, $\mathrm{CH}$ selects the $\mathrm{CM}$ node with the largest pheromone concentration on the link as IM_node. IM_ node is chosen as soon as there is data transmitted from $\mathrm{CH}$ to the sink node. $\mathrm{CM}$ is responsible for collecting and transmitting data to the $\mathrm{CH}$. The $\mathrm{CH}$ collects and merges the data and sends the converged data to the IM_node of the cluster, and the IM_node forwards the data to the sink node at a far distance. The choice of IM_node depends on node maximal pheromone concentration, and thus, 


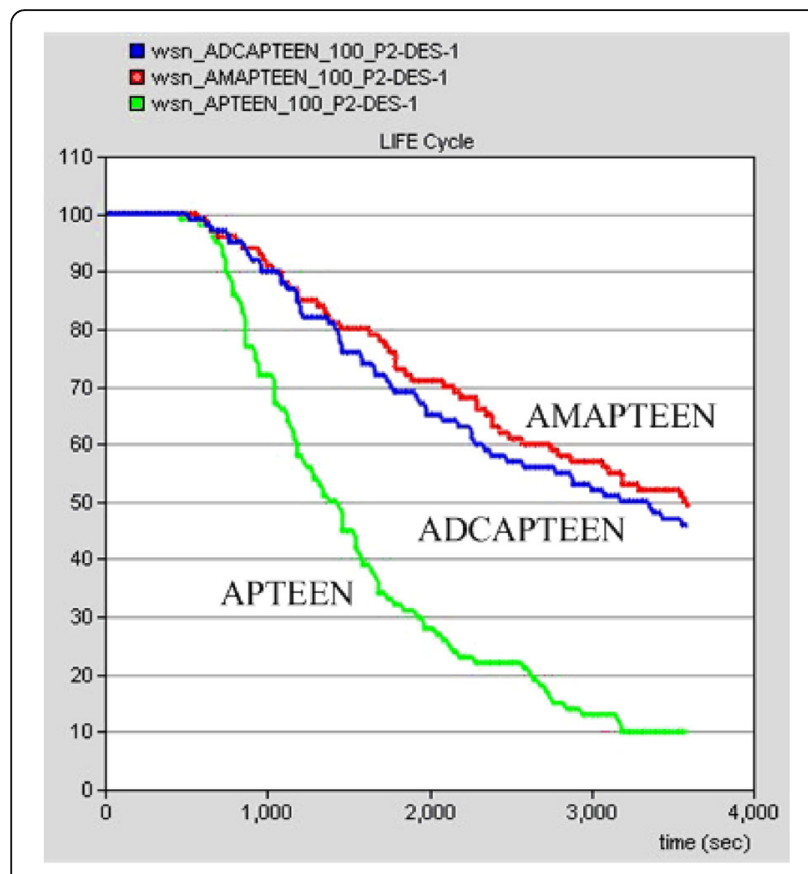

Fig. 15 The life cycle compared ADCAPTEEN with AMAPTEEN. This figure shows the comparison of network lifetime under ADCAPTEEN and AMAPTEEN. We can see that AMAPTEEN has further extended the network life cycle and improved the number of surviving nodes compared with ADCAPTEEN

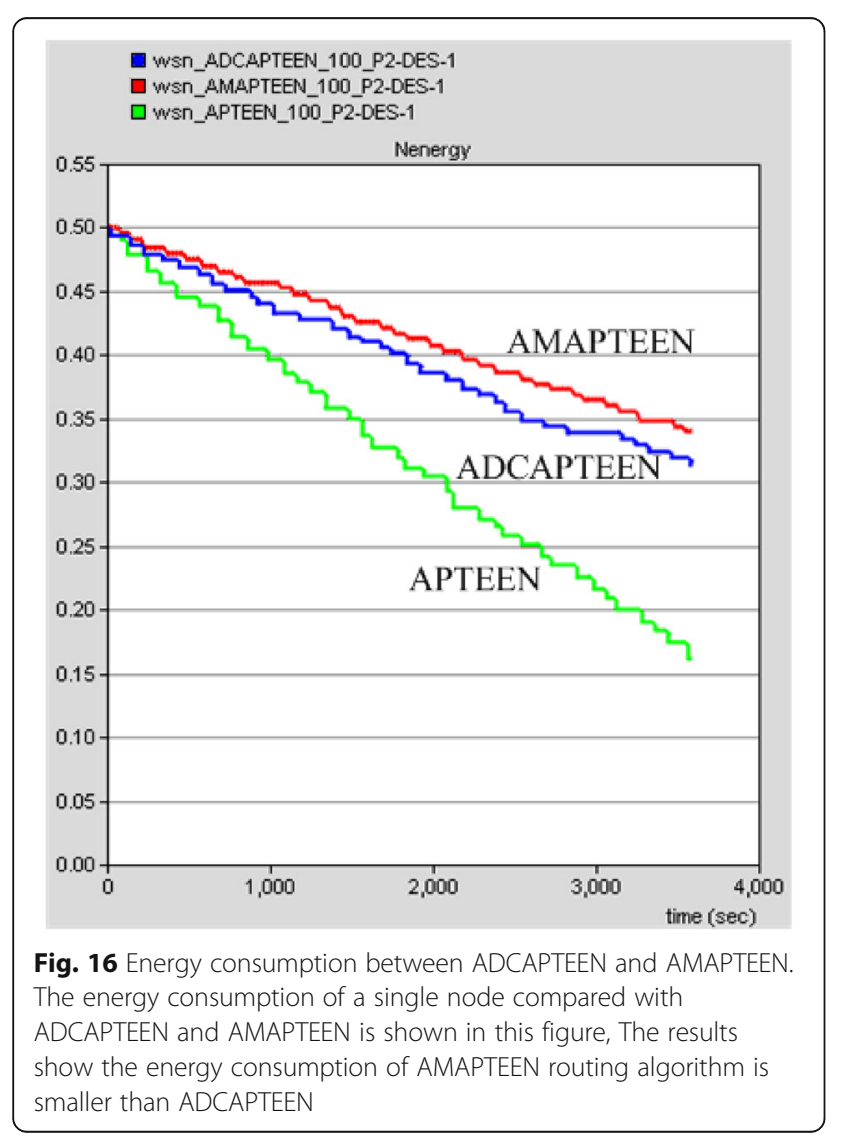

choosing the data transmission path is relatively optimal. And it is likely to be different, so there will be multiple data transfer paths from $\mathrm{CH}$ to sink. Streaming data to multiple paths, it reduces energy consumption, improves node survival rate, and extends life cycle of network further compared with ADCAPTEEN.

\section{Abbreviations}

ADCAPTEEN: Double cluster heads Adaptive Threshold-sensitive Energy Efficient Network based on ant colony; AMAPTEEN: Multiple Adaptive Threshold-sensitive Energy Efficient Network based on ant colony; APTEEN: Adaptive Threshold-sensitive Energy Efficient Network; CH: Cluster head; CM: Cluster member; CT: Count time; DAPTEEN: Distance Adaptive Threshold Sensitive Energy Efficient Sensor Network; DCCCA: Double cluster heads topology control algorithm based on the energy threshold which cater to heterogeneous wireless sensor network; DCH: Double cluster heads; DHSC: Double heads static cluster; EE-LEACH: Energy-efficient LEACH; HMAPTEEN: Hierarchal Multipath Adaptive Threshold-sensitive Energy Efficient Network; HT: Hard threshold; IM_node: Intermediate node;

LEACH: Low-Energy Adaptive Clustering Hierarchy; MCH: Master cluster head; ST: Soft threshold; SV: Sensed value; TEEN: Threshold-sensitive Energy Efficient Network; VCH: Vice cluster head; WSN: Wireless sensor network

\section{Funding}

This work was supported by the National Natural Science Foundation of China $(61761034,61261020)$ and the Natural Science Foundation of Inner Mongolia, China (2016MS0616).

\section{Availability of data and materials}

The materials and data are true and reliable in this paper.

\section{Authors' contributions}

W-SB (wangshubin@imu.edu.cn) is the corresponding author. M-JY and W-SB conceived the proposed scheme. M-JY conducted the detailed derivation to evaluate the performance of the proposed scheme and carried out the most experiments and data analysis and wrote the manuscript. MC and G-YH carries out the part experiments and data analysis, D-JT helped to improve the experimental simulation and polish the article language after the suggestions for revision of the manuscript, and W-SB reviewed the manuscript. All authors have read and approved the final manuscript.

\section{Competing interests}

The authors declare that they have no competing interests.

\section{Publisher's Note}

Springer Nature remains neutral with regard to jurisdictional claims in published maps and institutional affiliations.

\section{Author details}

${ }^{1}$ College of Electronic Information Engineering, Inner Mongolia University, Hohhot, China. ${ }^{2}$ Inner Mongolia University of Finance and Economics, Hohhot, China.

Received: 24 October 2017 Accepted: 18 April 2018 Published online: 03 May 2018

\section{References}

1. X Li, F Zhou, D Junping, LDTS: a lightweight and dependable trust system for clustered wireless sensor networks[]]. IEEE Trans. Inf. Forensics Sec. 8(6), 924-935 (2013)

2. Debabrata Singh, Sanjeet Kumar Nayak. Enhanced modified LEACH (EMODLEACH) protocol for WSN[J]. Int. Symp. Adv. Comput. Commun. (ISACC),328-333(2015). https://doi.org/10.1109/ISACC.2015.7377364

3. P Nayak, A Devulapalli, A fuzzy logic-based clustering algorithm for WSN to extend the network lifetime[J]. IEEE Sensors J. 16(1), 137-144 (2016)

4. Gopi Saminathan Arumugam, Thirumurugan Ponnuchamy. EE-LEACH: development of energy-efficient LEACH Protocol for data gathering in WSN[J]. EURASIP J. Wirel. Commun. Netw.,(1):1-9(2015). 
5. Krishnakumar Amirthalingam, Dr. Anuratha.Improved LEACH: A modified LEACH for wireless sensor network[J]. Int. Conf. Adv. Comput. Appl. (ICACA),255-258(2016). https://doi.org/10.1109/ICACA.2016.7887961

6. D Karaboga, S Okdem, C Ozturk, Cluster based wireless sensor network routing using artificial bee colony algorithm[J]. Wireless Network 18, 847-860 (2012)

7. Zubaria Andleeb, M.R. Anjum, M. Usman Sardar. Study the impact of multiple mobile sinks on lifetime of wireless sensor networks[J]. Int. Conf. Innovative Comput. Technol. (INTECH), 418-422(2016). https://doi.org/10. 1109/INTECH.2016.7845065

8. SD Muruganathan, AB Sesay, WA Krzymien, Analytical query response time evaluation for a two-level clustering hierarchy based wireless sensor network routing protocol[J]. IEEE Commun. Lett. 14(5), 486-488 (2011)

9. O Abouelkhair, A Elsaadany, Hierarchal energy efficient network protocol for wireless sensing[J]. Comput. Eng. Conf. (ICENCO), 128-132 (2014). https:// doi.org/10.1109/ICENCO.2014.7050444

10. Anjali, Anshul Garg, Suhali. Distance Adaptive Threshold Sensitive Energy Efficient Sensor Network (DAPTEEN) Protocol in WSN[J]. Signal Process. Comput. Control (ISPCC),114-119(2015). https://doi.org/10.1109/ISPCC.2015. 7375008

11. $X Y$ Yi, D Lu, A double heads static cluster algorithm for wireless sensor networks[J]. Environ. Sci. Inf. Appl. Technol. (ESIAT) 2, 635-638 (2010)

12. Q Xuegong, C Yan, A control algorithm based on double cluster-head for heterogeneous wireless sensor network[J]. Int. Conf. Ind. Inf. Syst. (IIS) 1, 541-544 (2010)

13. M Dorigo, V Maniezzo, A Colomi, The ant system: Optimizationby a Colony of cooperating agents [J]. IEEE Trans. Syst. Man Cybernetics B 26(2), 29-41 (1996)

14. D. Baghyalakshmi, J. Ebenezer, S. A. V. Satyamurty . Low latency and energy efficient routing protocols for wireless sensor networks[J]. International Conference on Wireless Communication and Sensor Computing (ICWCSC), 39(8):1-6 (2010)

\section{Submit your manuscript to a SpringerOpen ${ }^{\circ}$ journal and benefit from:}

- Convenient online submission

- Rigorous peer review

- Open access: articles freely available online

- High visibility within the field

- Retaining the copyright to your article

Submit your next manuscript at $>$ springeropen.com 